\title{
Correlation Functions as Nests of Self-Avoiding Paths
}

\author{
Nikolay M. Bogoliubov ${ }^{\star, \dagger}$, Cyril Malyshev ${ }^{\star, \dagger}$ \\ ${ }^{\star}$ St.-Petersburg Department of Steklov Institute of Mathematics, RAS \\ Fontanka 27, St.-Petersburg, RUSSIA \\ ${ }^{\dagger}$ ITMO University \\ Kronverksky 49, St.-Petersburg, RUSSIA
}

\begin{abstract}
We discuss connection between the $X X Z$ Heisenberg spin chain in the limiting case of zero anisotropy and some aspects of enumerative combinatorics. The representation of the Bethe wave functions via the Schur functions allows to apply the theory of symmetric functions to calculation of the correlation functions. We provide a combinatorial derivation of the dynamical correlation functions of the projection operator in terms of nests of self-avoiding lattice paths.
\end{abstract}

Key words: $X X Z$ Heisenberg spin chain; correlation functions; enumerative combinatorics 


\section{Introduction}

The theory of random walks, being one of the classical directions of enumerative combinatorics [1], was successfully applied in various fields: in the theory of quantum computations [2] and in the analysis of stock markets [3], in biology [4] and in psychology [5], in self-organized criticality [6] and in population processes [7].

The 'Random walks problem' in theoretical physics was first introduced by M. Fisher [8]. Fascinating connections to other research fields, such as Young diagrams, and the theory of random matrices, have been revealed one after another [9 14].

Some sections of enumerative combinatorics [1] and the theory of symmetric functions 15 has come to play an important role in the theory of integrable models [16, 17 and especially in the studies of correlation functions [18, 19. The aim of this paper is to represent correlation functions of $X X 0$ spin chain as sums over nests of self-avoiding lattice paths. The interpretation of correlation functions of bosonic integrable models in terms of random walks in multidimensional simplectical lattices was given in [20,21.

Two essentially different types of vicious walkers may be distinguished in classification of [8]. Suppose that there are $N$ walkers (particles) on a one-dimensional lattice. For the random turns model at each tick of the clock $d t$ only a single randomly chosen walker moves one step to the left or one step to the right while the rest are staying (Fig. 1). In the lock step version of the model at each tick of the clock each walker

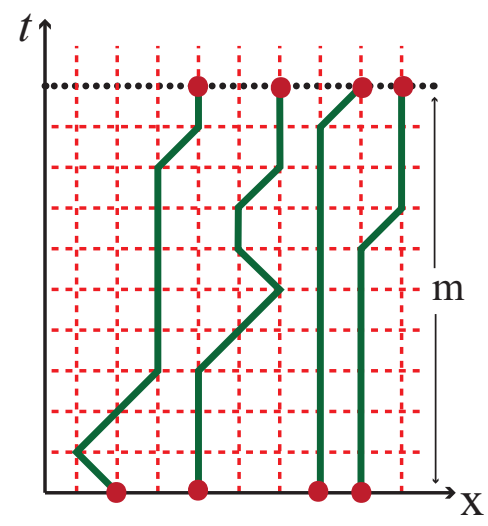

Figure 1: Random turns walkers.

moves to the left or to the right lattice site with equal probability (Fig. 2). Trajectories of random walkers can be viewed as directed lattice paths (i.e., the paths that cannot turn back) which start at sites say on the line $x$ and finish after $m$ steps on sites on the line $t=m$. Walkers are 'vicious' so that two or more walkers are prohibited to arrive at the same site simultaneously.

Random walks are closely related to plane partitions or three-dimensional Young diagrams. A plane partition is a two-dimensional array of nonnegative integers $n_{i, j}$ that are non-increasing both from left to right and from top to bottom: $n_{i, j} \geq n_{i, j+1}$ and $n_{i, j} \geq n_{i+1, j}$. Plane partitions may be represented as a stack of $n_{i, j}$ unit cubes above the point $(i, j)$ (Fig. 3).

Our paper is structured as follows. The current Section 1 is introductory. Section 2 


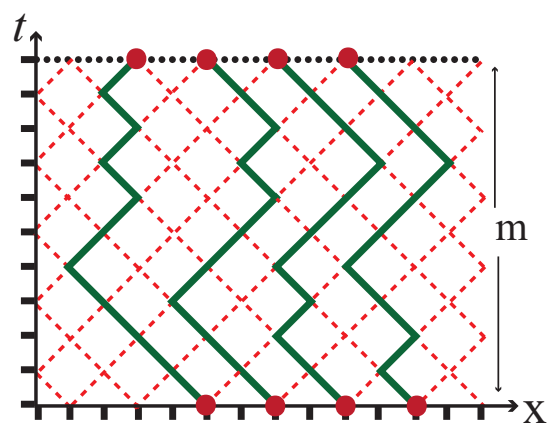

Figure 2: Lock step walkers.

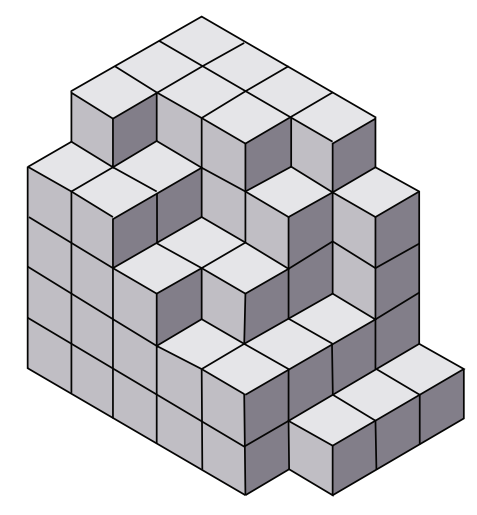

Figure 3: Plane partition.

is devoted to the free fermion limit of the $X X Z$ Heisenberg model. In Section 3 the correlation functions over zero particles ground state are discussed. In Section 4 the combinatorial description of the thermal correlation functions is given. In Section 5 it is shown that the thermal correlation function of the projection operator can be treated in terms of sums over nests of self-avoiding lattice paths of special type. An identity is obtained as a by-product which relates a trigonometric sum to an integer equal to a number of self-avoiding lattice paths. Discussion in Section 6 concludes the paper.

\section{$2 X X Z$ Heisenberg Spin Chain and Its Zero Anisotropy Limit}

The Heisenberg $X X Z$ model on the chain of $M+1$ sites is defined by the Hamiltonian

$$
H_{\mathrm{XXZ}}=-\frac{1}{2} \sum_{k=0}^{M}\left(\sigma_{k+1}^{-} \sigma_{k}^{+}+\sigma_{k+1}^{+} \sigma_{k}^{-}+\frac{\Delta}{2}\left(\sigma_{k+1}^{z} \sigma_{k}^{z}-\mathbb{I}\right)+\left(\sigma_{k}^{z}-\mathbb{I}\right)\right),
$$

where $\Delta \in \mathbb{R}$ is the anisotropy parameter. The local spin operators $\sigma_{k}^{ \pm}=\frac{1}{2}\left(\sigma_{k}^{x} \pm i \sigma_{k}^{y}\right)$ and $\sigma_{k}^{z}$ act nontrivially on $k^{\text {th }}$ site and obey the commutation rules:

$$
\left[\sigma_{k}^{+}, \sigma_{l}^{-}\right]=\delta_{k l} \sigma_{l}^{z}, \quad\left[\sigma_{k}^{z}, \sigma_{l}^{ \pm}\right]= \pm 2 \delta_{k l} \sigma_{l}^{ \pm}
$$


$\left(\delta_{k l}\right.$ is the Kronecker symbol). Besides, $\mathbb{I}$ acts in $(1)$ as identity operator at $k^{\text {th }}$ site. The spin operators act in the space $\mathfrak{H}_{M+1}$ spanned over the states $\bigotimes_{k=0}^{M}|s\rangle_{k}$, where $|s\rangle_{k}$ implies either spin "up", $|\uparrow\rangle$, or spin "down", $|\downarrow\rangle$, state at $k^{\text {th }}$ site. The states $|\uparrow\rangle \equiv\left(\begin{array}{l}1 \\ 0\end{array}\right)$ and $|\downarrow\rangle \equiv\left(\begin{array}{l}0 \\ 1\end{array}\right)$ provide a natural basis of the linear space $\mathbb{C}^{2}$. The state $|\Uparrow\rangle$ with all spins "up": $|\uparrow\rangle \equiv \bigotimes_{n=0}^{M}|\uparrow\rangle_{n}$ is annihilated by the Hamiltonian (1):

$$
H_{\mathrm{XXZ}}|\Uparrow\rangle=0 \text {. }
$$

The Hamiltonian (1) commutes with the operator $S^{z}$ of the third component of the total spin:

$$
\left[H_{\mathrm{XXZ}}, S^{z}\right]=0, \quad S^{z} \equiv \frac{1}{2} \sum_{k=0}^{M} \sigma_{k}^{z} .
$$

We shall consider the $X X$ Heisenberg model, which is the free fermion limit of the $X X Z$ Heisenberg spin chain. The Hamiltonian of the $X X$ spin chain arises as the zero anisotropy limit $\Delta \rightarrow 0$ of the Hamiltonian (1):

$$
H_{\mathrm{XX}}=\frac{1}{2} \mathcal{H}-\frac{1}{2} \sum_{k=0}^{M}\left(\sigma_{k}^{z}-\mathbb{I}\right)
$$

where $\mathcal{H}$ is the "hopping" part:

$$
\mathcal{H} \equiv-\sum_{k=0}^{M}\left(\sigma_{k+1}^{-} \sigma_{k}^{+}+\sigma_{k+1}^{+} \sigma_{k}^{-}\right) .
$$

The system described by the Hamiltonian (3) is of interest, for example, in the construction of the theory of quantum computations [22].

Consider an arbitrary state on a chain. It can be characterized by the number $N$ of spins "down" and the number $\mathcal{M} \equiv M-N+1$ of sites with spin "up". The $N$-particle state-vectors $\left|\Psi\left(\mathbf{u}_{N}\right)\right\rangle$, i.e., the states with $N$ spins "down", are convenient to express by means of the Schur functions:

$$
\left|\Psi\left(\mathbf{u}_{N}\right)\right\rangle=\sum_{\boldsymbol{\lambda} \subseteq\left\{\mathcal{M}^{N}\right\}} S_{\boldsymbol{\lambda}}\left(\mathbf{u}_{N}^{2}\right)\left(\prod_{k=1}^{N} \sigma_{\mu_{k}}^{-}\right)|\Uparrow\rangle .
$$

The sites with spin "down" states are labeled by the coordinates $\mu_{i}, 1 \leq i \leq N$. These coordinates constitute a strictly decreasing partition $\boldsymbol{\mu}=\left(\mu_{1}, \mu_{2}, \ldots, \mu_{N}\right)$, where the numbers $\mu_{i}$, called parts, respect the inequality $M \geq \mu_{1}>\mu_{2}>\ldots>\mu_{N} \geq 0$. The relation $\lambda_{j}=\mu_{j}-N+j$, where $1 \leq j \leq N$, connects the parts of $\boldsymbol{\lambda}$ to those of $\boldsymbol{\mu}$. Therefore, we can write: $\boldsymbol{\lambda}=\boldsymbol{\mu}-\boldsymbol{\delta}_{N}$, where $\boldsymbol{\delta}_{N}$ is the strict partition

$$
\boldsymbol{\delta}_{N} \equiv(N-1, N-2, \ldots, 1,0) .
$$

Besides, the bold notations like $\mathbf{u}_{N}^{2} \equiv\left(u_{1}^{2}, u_{2}^{2}, \ldots, u_{N}^{2}\right)$ imply sets of arbitrary complex numbers. The summation in (5) is over all partitions $\boldsymbol{\lambda}$ with parts satisfying $\mathcal{M} \geq$ $\lambda_{1} \geq \lambda_{2} \geq \cdots \geq \lambda_{N} \geq 0$. 
The Schur functions $S_{\boldsymbol{\lambda}}$ are defined by the Jacobi-Trudi relation:

$$
S_{\boldsymbol{\lambda}}\left(\mathbf{x}_{N}\right) \equiv S_{\boldsymbol{\lambda}}\left(x_{1}, x_{2}, \ldots, x_{N}\right) \equiv \frac{\operatorname{det}\left(x_{j}^{\lambda_{k}+N-k}\right)_{1 \leq j, k \leq N}}{\mathcal{V}\left(\mathbf{x}_{N}\right)},
$$

in which $\mathcal{V}\left(\mathbf{x}_{N}\right)$ is the Vandermonde determinant

$$
\mathcal{V}\left(\mathbf{x}_{N}\right) \equiv \operatorname{det}\left(x_{j}^{N-k}\right)_{1 \leq j, k \leq N}=\prod_{1 \leq m<l \leq N}\left(x_{l}-x_{m}\right)
$$

The conjugated state-vectors are given by

$$
\left\langle\Psi\left(\mathbf{v}_{N}\right)\right|=\sum_{\boldsymbol{\lambda} \subseteq\left\{\mathcal{M}^{N}\right\}}\langle\Uparrow|\left(\prod_{k=1}^{N} \sigma_{\mu_{k}}^{+}\right) S_{\boldsymbol{\lambda}}\left(\mathbf{v}_{N}^{-2}\right) .
$$

There is a natural correspondence between the coordinates of the spin "down" states $\boldsymbol{\mu}$ and the partition $\boldsymbol{\lambda}$ expressed by the Young diagram (see Fig. 4 ).

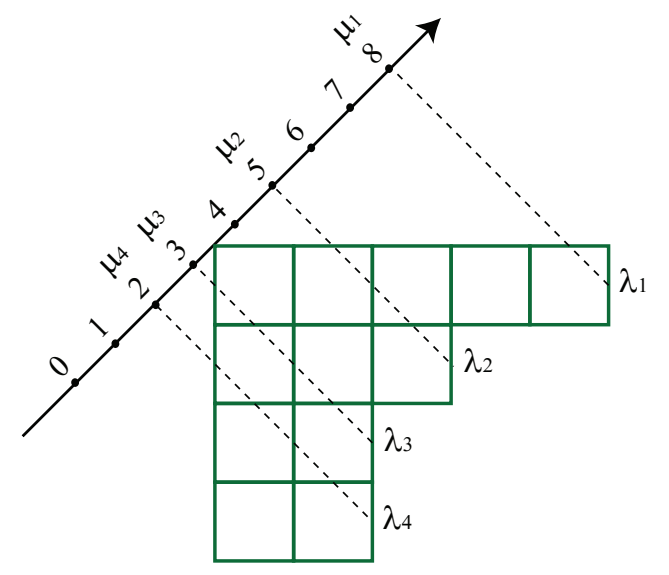

Figure 4: Relation of the spin "down" coordinates $\boldsymbol{\mu}=(8,5,3,2)$ and partition $\boldsymbol{\lambda}=$ $(5,3,2,2)$ for $M=8, N=4$.

Assume that the periodic boundary conditions are imposed: $\sigma_{k+(M+1)}^{\#}=\sigma_{k}^{\#}$. If the parameters $u_{j}^{2} \equiv e^{i \theta_{j}}(1 \leq j \leq N)$ satisfy the Bethe equations,

$$
e^{i(M+1) \theta_{j}}=(-1)^{N-1}, \quad 1 \leq j \leq N,
$$

then the state-vectors (5) become the eigen-vectors of the Hamiltonian (3) [17]:

$$
H_{\mathrm{XX}}\left|\Psi\left(\boldsymbol{\theta}_{N}\right)\right\rangle=E_{N}\left(\boldsymbol{\theta}_{N}\right)\left|\Psi\left(\boldsymbol{\theta}_{N}\right)\right\rangle .
$$

The solutions $\theta_{j}$ to the Bethe equations 10 can be parameterized so that

$$
\theta_{j}=\frac{2 \pi}{M+1}\left(I_{j}-\frac{N-1}{2}\right), \quad 1 \leq j \leq N
$$


where $I_{j}$ are integers or half-integers depending on whether $N$ is odd or even.

The eigen-energies in (11) are equal to

$$
E_{N}\left(\boldsymbol{\theta}_{N}\right)=N-\sum_{j=1}^{N} \cos \theta_{j}=N-\sum_{j=1}^{N} \cos \left(\frac{2 \pi}{M+1}\left(I_{j}-\frac{N-1}{2}\right)\right) .
$$

The ground state of the model is the eigen-state that corresponds to the lowest eigenenergy $E_{N}\left(\boldsymbol{\theta}_{N}^{\mathrm{g}}\right)$. It is determined by the solution to the Bethe equations $(12)$ at $I_{j}=$ $N-j$ :

$$
\theta_{j}^{\mathrm{g}} \equiv \frac{2 \pi}{M+1}\left(\frac{N+1}{2}-j\right), \quad 1 \leq j \leq N
$$

and is equal to

$$
E_{N}\left(\boldsymbol{\theta}_{N}^{\mathrm{g}}\right)=N-\frac{\sin \frac{\pi N}{M+1}}{\sin \frac{\pi}{M+1}} .
$$

In this paper we shall be restricted with the case of finite size of the system in order to consider the dynamical correlation function called the persistence of ferromagnetic string and related to the projection operator $\bar{\Pi}_{n}$ that forbids spin "down" states on $n$ consecutive sites of the chain [18]:

$$
\mathcal{T}\left(\boldsymbol{\theta}_{N}^{\mathrm{g}}, n, t\right) \equiv \frac{\left\langle\Psi\left(\boldsymbol{\theta}_{N}^{\mathrm{g}}\right)\left|\bar{\Pi}_{n} e^{-t H_{\mathrm{Xx}}} \bar{\Pi}_{n}\right| \Psi\left(\boldsymbol{\theta}_{N}^{\mathrm{g}}\right)\right\rangle}{\left\langle\Psi\left(\boldsymbol{\theta}_{N}^{\mathrm{g}}\right)\left|e^{-t H_{\mathrm{Xx}}}\right| \Psi\left(\boldsymbol{\theta}_{N}^{\mathrm{g}}\right)\right\rangle}, \quad \bar{\Pi}_{n} \equiv \prod_{j=0}^{n-1} \frac{\mathbb{I}+\sigma_{j}^{z}}{2}
$$

where $t \in \mathbb{C}$. We assume that $\bar{\Pi}_{0}$ is the identity operator so that $\mathcal{T}\left(\boldsymbol{\theta}_{N}^{\mathrm{g}}, 0, \beta\right)=1$.

\section{Correlations Over Zero Particles Ground State}

First, we shall consider the simplest one-particle correlation function

$$
G(j, m \mid t) \equiv\left\langle\Uparrow\left|\sigma_{j}^{+} e^{-\frac{t}{2} \mathcal{H}} \sigma_{m}^{-}\right| \Uparrow\right\rangle,
$$

where $\mathcal{H}$ is the Hamiltonian (4), which can be re-expressed through, so-called, hopping matrix $\boldsymbol{\Delta} \equiv\left(\Delta_{n m}\right)_{0 \leq n, m \leq M} \mid 14,23$. In the problem of vicious walkers it is more appropriate to use $\mathcal{H}$ expressed as follows:

$$
\mathcal{H}=-\sum_{n, m=0}^{M} \Delta_{n m} \sigma_{n}^{-} \sigma_{m}^{+}, \quad \Delta_{n m} \equiv \delta_{|n-m|, 1}+\delta_{|n-m|, M} .
$$

Differentiating $G(j, m \mid t)$ with respect to $t$ and applying the commutation relation

$$
\left[\mathcal{H}, \sigma_{m}^{-}\right]=-\sum_{n=0}^{M} \Delta_{n m} \sigma_{n}^{-} \sigma_{m}^{z}
$$

we obtain, 14, 23], the difference-differential equation:

$$
\frac{d}{d t} G(j, m \mid t)=\frac{1}{2}(G(j, m-1 \mid t)+G(j, m+1 \mid t)) .
$$


Equation 19$)$ is supplied at fixed $j$ with the periodicity requirement $G(j, m+M+1 \mid t)=$ $G(j, m \mid t)$. An analogous requirement $G(j+M+1, m \mid t)=G(j, m \mid t)$ is valid for fixed $m$ as well. Besides, the "initial condition" is given by $G(j, m \mid 0)=\delta_{j m}$.

The correlator $G(j, m \mid t)$ (16) may be considered as the exponential generating function of random walks. Indeed, let us introduce the notation $\mathcal{D}_{\lambda}^{K}$ for the operator of differentiation of $K$-th order with respect to $\lambda$ at the point $\lambda=0$. Let us put the correlation function (16) as a series in powers of $t$ :

$$
G(j, m \mid t)=\sum_{K=0}^{\infty} \frac{(t / 2)^{K}}{K !} \mathfrak{G}(j, m \mid K) .
$$

Acting by $\mathcal{D}_{t / 2}^{K}$ on $G(j, m \mid t) \sqrt{16}$ one obtains the coefficients $\mathfrak{G}(j, m \mid K)$ which are reduced to the entries of the $K^{\text {th }}$ power of $\boldsymbol{\Delta}(17)$ :

$$
\begin{array}{r}
\mathfrak{G}(j, m \mid K)=\mathcal{D}_{t / 2}^{K} G(j, m \mid t) \\
=\left\langle\Uparrow\left|\sigma_{j}^{+}(-\mathcal{H})^{K} \sigma_{m}^{-}\right| \Uparrow\right\rangle=\left(\Delta^{K}\right)_{j m} .
\end{array}
$$

Indeed, applying the commutation relation (18), one obtains:

$$
(-\mathcal{H})^{K} \sigma_{m}^{-}|\Uparrow\rangle=\sum_{n=0}^{M}\left(\Delta^{K}\right)_{n m} \sigma_{n}^{-}|\Uparrow\rangle
$$

Equation (22) may be interpreted in the following way. Position of the walker on the chain is labelled by the spin "down" state, while the spin "up" states correspond to empty sites. Each matrix $\boldsymbol{\Delta}$ in the product 22 corresponds to a transition between two neighboring sites. The relation $(22)$ enables to enumerate all admissible paths of the walker starting from the $m^{\text {th }}$ site. The state $\langle\Uparrow| \sigma_{j}^{+}$acting on 22 from left allows to fix the ending point of the paths because of the orthogonality of the spin states, and Eq. (21) thus arises.

Let $\left|P_{K}(m \rightarrow j)\right|$ to denote the number of paths between the $m^{\text {th }}$ and $j^{\text {th }}$ sites. It is clear that $\mathfrak{G}(j, m \mid K) \equiv\left|P_{K}(m \rightarrow j)\right|=\left(\Delta^{K}\right)_{j m}$, and the generating function $G(j, m \mid t)$ includes processes with all possible numbers of steps. It follows from Eq. (21) that $\mathfrak{G}(j, m \mid K)$ satisfies the equation:

$$
\mathfrak{G}(j, m \mid K+1)=\mathfrak{G}(j, m-1 \mid K)+\mathfrak{G}(j, m+1 \mid K),
$$

with the "initial" condition $\mathfrak{G}(j, m \mid 0)=\delta_{j m}$.

In theory of lattice paths the following generating function is usually used:

$$
F(j, m \mid z)=\sum_{K=0}^{\infty} z^{K}\left\langle\Uparrow\left|\sigma_{j}^{+}(-\mathcal{H})^{K} \sigma_{m}^{-}\right| \Uparrow\right\rangle \equiv \sum_{K=0}^{\infty} z^{K} \mathfrak{G}(j, m \mid K),
$$

which is the Laplace transform of the exponential generating function (16):

$$
\int_{0}^{\infty} G(j, m \mid t) e^{-\frac{t}{z}} d t=z\left\langle\Uparrow\left|\sigma_{j}^{+}\left(\frac{1}{1+z \mathcal{H}}\right) \sigma_{m}^{-}\right| \Uparrow\right\rangle=z F(j, m \mid z), \quad \Re z>0 .
$$


Consider now the multi-particle correlation function

$$
G(\mathbf{j} ; \mathbf{l} \mid t)=\left\langle\Uparrow\left|\left(\prod_{n=1}^{N} \sigma_{j_{n}}^{+}\right) e^{-\frac{t}{2} \mathcal{H}}\left(\prod_{k=1}^{N} \sigma_{l_{k}}^{-}\right)\right| \Uparrow\right\rangle
$$

which is parametrized by multi-indices $\mathbf{j} \equiv\left(j_{1}, j_{2}, \ldots, j_{N}\right)$ and $\mathbf{l} \equiv\left(l_{1}, l_{2}, \ldots, l_{N}\right)$. This correlator is the generation function of $N$ random turns vicious walkers (see Fig. 1). Really, let $\left|P_{K}(\mathbf{j} \rightarrow \mathbf{l})\right|$ be the number of $K$-edge paths traced by $N$ vicious walkers in the random turns model. The commutation relation

$$
\left[\mathcal{H}, \sigma_{l_{1}}^{-} \sigma_{l_{2}}^{-} \ldots \sigma_{l_{N}}^{-}\right]=\sum_{k=1}^{N} \sigma_{l_{1}}^{-} \ldots \sigma_{l_{k-1}}^{-}\left[\mathcal{H}, \sigma_{l_{k}}^{-}\right] \sigma_{l_{k+1}}^{-} \ldots \sigma_{l_{N}}^{-}
$$

enables us to see that the average

$$
\mathfrak{G}(\mathbf{j} ; \mathbf{l} \mid K) \equiv \mathcal{D}_{t / 2}^{K} G(\mathbf{j} ; \mathbf{l} \mid t)=\left\langle\Uparrow\left|\left(\prod_{n=1}^{N} \sigma_{j_{n}}^{+}\right)(-\mathcal{H})^{K}\left(\prod_{k=1}^{N} \sigma_{l_{k}}^{-}\right)\right| \Uparrow\right\rangle
$$

is equal to the number $\left|P_{K}(\mathbf{j} \rightarrow \mathbf{l})\right|$ of configurations of $N$ random turns walkers being initially located on the sites $l_{1}>l_{2}>\cdots>l_{N}$ and arrived after $K$ steps at the positions $j_{1}>j_{2}>\cdots>j_{N}$ :

$$
\left|P_{K}(\mathbf{j} \rightarrow \mathbf{l})\right|=\mathfrak{G}(\mathbf{j} ; \mathbf{l} \mid K)
$$

The condition that vicious walkers do not touch each other up to $N$ steps is guaranteed by the property of the Pauli matrices $\left(\sigma_{k}^{ \pm}\right)^{2}=0$.

Differentiating (26) by $t$ and applying (27) we obtain for fixed $\mathbf{j}$ the equation

$$
\begin{array}{r}
\frac{d}{d t} G(\mathbf{j} ; \mathbf{l} \mid t)=\frac{1}{2} \sum_{k=1}^{N}\left(G\left(\mathbf{j} ; l_{1}, l_{2}, \ldots, l_{k}+1, \ldots, l_{N} \mid t\right)\right. \\
\left.+G\left(\mathbf{j} ; l_{1}, l_{2}, \ldots, l_{k}-1, \ldots, l_{N} \mid t\right)\right)
\end{array}
$$

(and a similar one for fixed $\mathbf{l}$ ). The non-intersection condition means that $G(\mathbf{j} ; \mathbf{l} \mid t)=0$ if $l_{k}=l_{p}\left(\right.$ or $\left.j_{k}=j_{p}\right)$ for any $1 \leq k, p \leq N$. The "initial condition" is: $G(\mathbf{j} ; \mathbf{l} \mid 0)=$ $\prod_{m=1}^{N} \delta_{j_{m}, l_{m}}$.

The generating function $G(\mathbf{j} ; \mathbf{l} \mid t)$ respecting Eq. 30 is given by the following

Proposition. Solution to Eq. (30) takes the form:

$$
G(\mathbf{j} ; \mathbf{l} \mid t)=\frac{e^{t N}}{(M+1)^{N}} \sum_{\left\{\boldsymbol{\phi}_{N}\right\}} e^{-t E_{N}\left(\boldsymbol{\phi}_{N}\right)}\left|\mathcal{V}\left(e^{i \phi_{N}}\right)\right|^{2} S_{\boldsymbol{\lambda}^{L}}\left(e^{i \phi_{N}}\right) S_{\boldsymbol{\lambda}^{R}}\left(e^{-i \boldsymbol{\phi}_{N}}\right)
$$

where the strict partitions $\mathbf{j}$ and $\mathbf{l}$ and the partitions $\boldsymbol{\lambda}^{L}$ and $\boldsymbol{\lambda}^{R}$ are related: $\boldsymbol{\lambda}^{L}=\mathbf{j}-\boldsymbol{\delta}_{N}$, $\boldsymbol{\lambda}^{R}=\mathbf{l}-\boldsymbol{\delta}_{N}$. The eigen-energy $E_{N}\left(\boldsymbol{\phi}_{N}\right)$ is defined by $(13), e^{ \pm i \phi} \equiv\left(e^{ \pm i \phi_{1}}, e^{ \pm i \phi_{2}}, \ldots, e^{ \pm i \phi_{N}}\right)$, and $\mathcal{V}\left(e^{i \phi_{N}}\right)$ is defined by (8).

Proof. It is easy to verify that the solution of 30 is given by

$$
G(\mathbf{j} ; \mathbf{l} \mid t)=\operatorname{det}\left(G\left(j_{r}, l_{s} \mid t\right)\right)_{1 \leq r, s \leq N}
$$


where $G(j, l \mid t)$ is the one-particle generating function (16) satisfying Eq. (19). The solution 32 may be expressed in the form:

$$
G(\mathbf{j} ; \mathbf{l} \mid t)=\frac{1}{(M+1)^{N} N !} \sum_{s_{1}, \ldots, s_{N}=0}^{M} e^{t \sum_{m=1}^{N} \cos \phi_{s_{m}}} \operatorname{det}\left(e^{i\left(j_{r}-l_{s}\right) \phi_{s_{r}}}\right)_{1 \leq r, s \leq N}
$$

where the parametrization is the same as in (31). The antisymmetry of the summand with respect to permutations of $\phi_{1}, \ldots, \phi_{N}$ enables to transform $\operatorname{det}\left(e^{i\left(j_{r}-l_{s}\right) \phi_{s_{r}}}\right)_{1 \leq r, s \leq N}$ in (33) into the product of $\operatorname{det}\left(e^{i j_{r} \phi_{s_{r}}}\right)_{1 \leq r, s \leq N}$ and $\operatorname{det}\left(e^{-i l_{s} \phi_{s_{r}}}\right)_{1 \leq r, s \leq N}$. So right-hand side of (33) is expressed in terms of the Schur functions (7), and the representation (31) is thus valid. It is clear that Eq. (31) at $N=1$ gives the solution to (19).

Corollary. From Eqs. (28) and $(31)$ we obtain that $\mathfrak{G}(\mathbf{j} ; \mathbf{l} \mid K)$ is represented as the trigonometric sum:

$$
\begin{aligned}
\mathfrak{G}(\mathbf{j} ; \mathbf{l} \mid K) & =\frac{1}{(M+1)^{N}} \sum_{\left\{\boldsymbol{\phi}_{N}\right\}}\left(2 \sum_{m=1}^{N} \cos \phi_{m}\right)^{K} \\
& \times\left|\mathcal{V}\left(e^{i \phi_{N}}\right)\right|^{2} S_{\boldsymbol{\lambda}^{L}}\left(e^{i \boldsymbol{\phi}_{N}}\right) S_{\boldsymbol{\lambda}^{R}}\left(e^{-i \phi_{N}}\right),
\end{aligned}
$$

which takes according to $(29)$ the integer value $\left|P_{K}(\mathbf{j} \rightarrow \mathbf{l})\right|$.

In the particular case when $\mathbf{j}=\mathbf{l}=\boldsymbol{\delta}_{N}$, where $\boldsymbol{\delta}_{N}$ is defined by (6), Eq. (34) is specified as

$$
\mathfrak{G}\left(\boldsymbol{\delta}_{N} ; \boldsymbol{\delta}_{N} \mid K\right)=\frac{1}{(M+1)^{N}} \sum_{\left\{\phi_{N}\right\}}\left(2 \sum_{m=1}^{N} \cos \phi_{m}\right)^{K}\left|\mathcal{V}\left(e^{i \phi_{N}}\right)\right|^{2}
$$

since $\boldsymbol{\lambda}^{L}=\boldsymbol{\lambda}^{R}=(0,0, \ldots, 0)$ and so

$$
S_{(0,0, \ldots, 0)}\left(e^{i \phi_{N}}\right)=S_{(0,0, \ldots, 0)}\left(e^{-i \phi_{N}}\right)=1 .
$$

In the thermodynamic limit the sum (35) becomes the Gross-Witten partition function 25 and expresses, as well, the distribution of the length of the longest increasing subsequence of random permutations [26].

\section{Lattice Paths Interpretations of the Determinantal Representations}

In this section an algebraic approach to the calculation of the correlation functions $\mathcal{T}\left(\boldsymbol{\theta}_{N}^{\mathrm{g}}, n, t\right)$ 15 is developed. The approach is based on the Cauchy-Binet formula for the Schur functions:

$$
\mathcal{P}_{L-n}(\mathbf{y}, \mathbf{x}) \equiv \sum_{\boldsymbol{\lambda} \backslash \mathbf{n} \subseteq\left\{(L-n)^{N}\right\}} S_{\boldsymbol{\lambda}}(\mathbf{x}) S_{\boldsymbol{\lambda}}(\mathbf{y})=\left(\prod_{l=1}^{N} y_{l}^{n} x_{l}^{n}\right) \frac{\operatorname{det}_{N \times N} T(\mathbf{x}, \mathbf{y})}{\mathcal{V}_{N}(\mathbf{x}) \mathcal{V}_{N}(\mathbf{y})}
$$


where $\sum_{\boldsymbol{\lambda} \backslash \mathbf{n} \subseteq\left\{(L-n)^{N}\right\}}$ implies summation over all non-strict partitions $\boldsymbol{\lambda}$ with the parts satisfying the inequality:

$$
L-n \geq \lambda_{1}-n \geq \lambda_{2}-n \geq \cdots \geq \lambda_{N}-n \geq 0 .
$$

The entries $\left(T_{k j}(\mathbf{x}, \mathbf{y})\right)_{1 \leq k, j \leq N}$ of the $N \times N$ matrix $T(\mathbf{x}, \mathbf{y})$ in right-hand side of (36) are expressed as

$$
T_{k j}=\frac{1-\left(x_{k} y_{j}\right)^{L-n+N}}{1-x_{k} y_{j}} .
$$

The scalar products of the state-vectors, as well as the correlation functions, are connected with the generating functions of boxed plane partitions [19]. To study the asymptotical behaviour of the introduced correlation functions, we need the CauchyBinet relation (36) taken in the $q$-parameterization

$$
\mathbf{y}=\mathbf{q} \equiv\left(q, q^{2}, \ldots, q^{N}\right), \quad \mathbf{x}=\mathbf{q} / q \equiv\left(1, q, \ldots, q^{N-1}\right) .
$$

Letting $L=\mathcal{M}$, we obtain from $(36)$ :

$$
\begin{aligned}
& \sum_{\boldsymbol{\lambda} \backslash \mathbf{n} \subseteq\left\{(\mathcal{M}-n)^{N}\right\}} S_{\boldsymbol{\lambda}}(\mathbf{q}) S_{\boldsymbol{\lambda}}(\mathbf{q} / q) \\
= & \frac{q^{n N^{2}}}{\mathcal{V}_{N}(\mathbf{q}) \mathcal{V}_{N}(\mathbf{q} / q)} \operatorname{det}\left(\frac{1-q^{(M+1-n)(j+k-1)}}{1-q^{j+k-1}}\right)_{1 \leq j, k \leq N} \\
= & q^{n N^{2}} q^{\frac{N(\mathcal{M}-n)}{2}(1-\mathcal{M}+n)} \operatorname{det}\left(\left[\begin{array}{c}
2 N+i-1 \\
N+j-1
\end{array}\right]\right)_{1 \leq i, j \leq \mathcal{M}-n} \\
= & q^{n N^{2}} Z_{q}(N, N, \mathcal{M}-n) .
\end{aligned}
$$

The entries in $(38)$ are the $q$-binomial coefficients defined as

$$
\left[\begin{array}{l}
R \\
r
\end{array}\right] \equiv \frac{[R] !}{[r] ![R-r] !}, \quad[n] \equiv \frac{1-q^{n}}{1-q} .
$$

Besides, $Z_{q}(N, N, \mathcal{M}-n)$ in $(39)$ is the MacMahon generating function of plane partitions in the box $\mathcal{B}(N, N, \mathcal{M}-n)$ of size $N \times N \times(\mathcal{M}-n)$ :

$$
Z_{q}(N, N, \mathcal{M}-n)=\prod_{k=1}^{N} \prod_{j=1}^{N} \frac{1-q^{\mathcal{M}-n+j+k-1}}{1-q^{j+k-1}} .
$$

The number of plane partitions in $\mathcal{B}(N, N, \mathcal{M}-n)$ is obtained from (41) at $q \rightarrow 1$ and is equal to

$$
A(N, N, \mathcal{M}-n)=\prod_{k=1}^{N} \prod_{j=1}^{N} \frac{\mathcal{M}-n+j+k-1}{j+k-1} .
$$

A combinatorial description of the Schur functions may be given in terms of semistandard Young tableaux [24], which are in one-to-one correspondence with the nests of 
self-avoiding lattice paths. A semi-standard Young tableau $\mathrm{T}$ of shape $\boldsymbol{\lambda}$ is a diagram whose cells are filled with positive integers $n \in \mathbb{N}$ weakly increasing along rows and strictly increasing along columns. Provided a semi-standard tableau $\mathrm{T}$ is given, the corresponding Schur function is defined as

$$
S_{\boldsymbol{\lambda}}\left(x_{1}, x_{2}, \ldots, x_{m}\right)=\sum_{\{\mathrm{T}\}} \mathbf{x}^{\top}, \quad \mathbf{x}^{\top} \equiv \prod_{i, j} x_{\boldsymbol{T}_{i j}}
$$

where the monomial $\mathbf{x}^{\top}$ is the weight equal to the product over all entries $\mathrm{T}_{i j}(i$ and $j$ label rows and columns of tableau $T$ ). The sum in (43) is over all tableaux $T$ of shape $\boldsymbol{\lambda}_{N}$ with the entries taken from the set $[m] \equiv\{1,2, \ldots, m\}, m \geq N$.

There is a natural way of representing each semi-standard tableau of shape $\boldsymbol{\lambda}$ with entries not exceeding $N$ as a nest of self-avoiding lattice paths with prescribed start and end points. Let $T_{i j}$ be an entry in $i^{\text {th }}$ row and $j^{\text {th }}$ column of tableau $T$. The $i^{\text {th }}$ lattice path (counted from the top of $T$ ) encodes the $i^{\text {th }}$ row of the tableau $(i=1, \ldots, N)$. A nest $\mathcal{C}$ consists of paths going from points $C_{i}=(i, N-i)$ to points $\left(N, \mu_{i}=\lambda_{i}+N-i\right)$ (see Fig. 5). Each path makes $\lambda_{i}$ steps to the north so that the steps along the line $x_{j}$ correspond to occurrences of the letter $j$ in the tableau $T$. The power $l_{j}$ of $x_{j}$ in the weight of any particular nest of paths is the number of steps to north taken along the vertical line $x_{j}$. Thus, an equivalent representation of the Schur function is

$$
S_{\boldsymbol{\lambda}}\left(x_{1}, x_{2}, \ldots, x_{N}\right)=\sum_{\{\mathcal{C}\}} \prod_{j=1}^{N} x_{j}^{l_{j}},
$$

where summation is over all admissible nests $\mathcal{C}$.

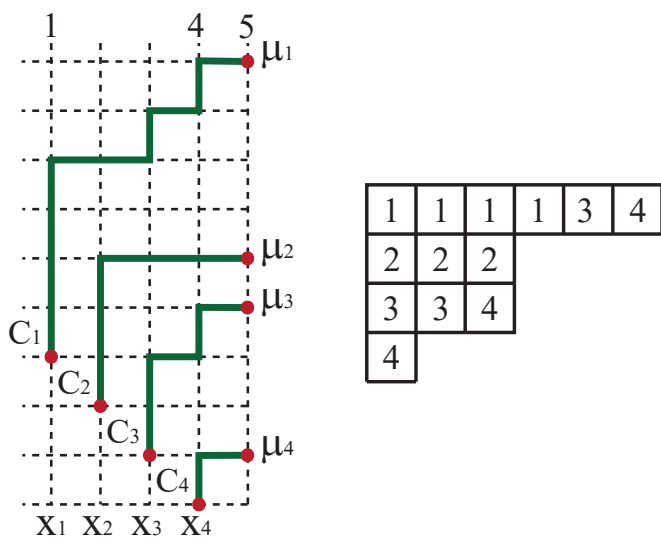

Figure 5: A semistandard tableau of shape $\boldsymbol{\lambda}=(6,3,3,1)$ as a nest $\mathcal{C}$ of lattice paths. The weight of $\mathcal{C}$ is $x_{1}^{4} x_{2}^{3} x_{3}^{3} x_{4}^{3}$.

From (44) it follows that the number of the described nests of paths is

$$
S_{\boldsymbol{\lambda}}(\mathbf{1})=\sum_{\{\mathcal{C}\}} 1=\prod_{1 \leq j<k \leq K} \frac{\lambda_{j}-j-\lambda_{k}+k}{k-j}=\prod_{1 \leq j<k \leq K} \frac{\mu_{j}-\mu_{k}}{k-j},
$$


where $S_{\boldsymbol{\lambda}}(\mathbf{1}) \equiv S_{\boldsymbol{\lambda}}(1,1, \ldots, 1),[15]$.

The $k^{\text {th }}$ lattice path is contained within a rectangle of the size $\lambda_{k} \times(N-k)$, $1 \leq k \leq N$. The starting point of each path is the lower left vertex. We define the volume of the path as the number of cells below the path within the corresponding rectangle. The volume of the nest of lattice paths $\mathcal{C}$ is equal to the volume of lattice paths:

$$
|\zeta|_{\mathcal{C}}=\sum_{j=1}^{N}(N-j) l_{j}=\sum_{j=1}^{N}(j-1) l_{N-j+1} .
$$

It follows that the $q$-parametrized Schur function is a partition function of the described nest:

$$
S_{\boldsymbol{\lambda}}(\mathbf{q})=\sum_{\{\mathcal{C}\}} q^{|\xi| \mathcal{C}}=q^{|\boldsymbol{\lambda}|} \sum_{\{\mathcal{C}\}} q^{|\zeta| \mathcal{C}},
$$

where $|\boldsymbol{\lambda}|=\sum_{k=1}^{N} \lambda_{k}$ is the weight of partition.

The Schur function corresponding to the conjugate nest of self-avoiding paths is equal to

$$
S_{\boldsymbol{\lambda}}\left(y_{1}, y_{2}, \ldots, y_{N}\right)=\sum_{\{\mathcal{B}\}} \prod_{j=1}^{N} y_{j}^{M-l_{j}},
$$

where summation is over all admissible nests $\mathcal{B}$ of $N$ self-avoiding lattice paths (see Fig. 6). The volume of the nest $\mathcal{B}$ is given by

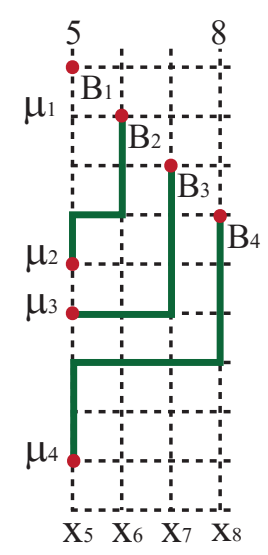

Figure 6: Conjugated nest $\mathcal{B}$ of lattice paths.

$$
|\zeta|_{\mathcal{B}}=\sum_{j=1}^{N}(j-1)\left(M-l_{j}\right),
$$

and the partition function of $\mathcal{B}$ (see Fig. 6) is obtained from (48) in the parametrization $\mathbf{y}=\mathbf{q}_{N} / q$ :

$$
S_{\boldsymbol{\lambda}}\left(\frac{\mathbf{q}_{N}}{q}\right)=\sum_{\{\mathcal{B}\}} q^{\sum_{j=1}^{N}(j-1)\left(\mathcal{M}-b_{j}\right)},
$$


where Eq. 49 is used, and summation is over all admissible nests $\mathcal{B}$ of $N$ self-avoiding lattice paths.

The scalar product, being the product of two Schur functions, may be graphically expressed as a nest of $N$ self-avoiding lattice paths starting at the equidistant points $C_{i}$ and terminating at the equidistant points $B_{i}(1 \leq i \leq N)$. This configuration, known as a watermelon, is presented in Fig. 7. The scalar product is given by the sum of all such watermelons. Rotating Fig. 7 by $\frac{\pi}{4}$ counter-clockwise we see that the watermelon configuration is a particular case of configuration of paths for lock step random walkers (see Fig. 2).

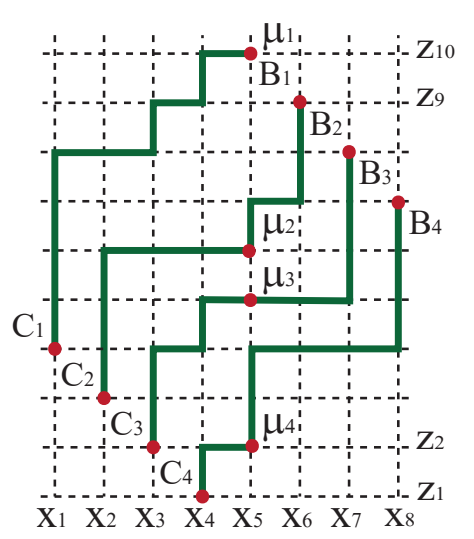

(A)

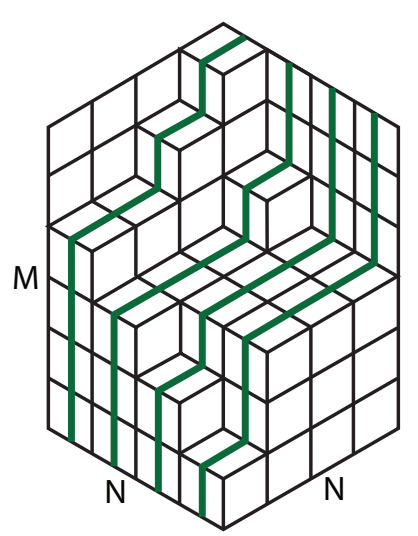

(B)

Figure 7: Watermelon configuration and correspondent plane partition.

Using definitions (5) and (9) enables one to obtain the $q$-parameterized average of the projection operator $\bar{\Pi}_{n}(15)$ (see $(39)$ ):

$$
\begin{aligned}
& \left\langle\Psi_{N}\left(\mathbf{q}^{-\frac{1}{2}}\right)\left|\bar{\Pi}_{n}\right| \Psi_{N}\left((\mathbf{q} / q)^{\frac{1}{2}}\right)\right\rangle \\
& =\sum_{\boldsymbol{\lambda} \backslash \mathbf{n} \subseteq\left\{(\mathcal{M}-n)^{N}\right\}} S_{\boldsymbol{\lambda}}(\mathbf{q}) S_{\boldsymbol{\lambda}}(\mathbf{q} / q)=q^{n N^{2}} Z(N, N, \mathcal{M}-n) .
\end{aligned}
$$

The partition function of watermelons with the end points $C_{i}, B_{i}, 1 \leq i \leq N$ (the generating function of watermelons) is given by (51) at $n=0$. 


\section{Correlations Over $N$-Particles Ground State}

The transition amplitude

$$
\begin{aligned}
& \left\langle\Psi\left(\mathbf{v}_{N}\right)\left|\bar{\Pi}_{n} e^{-\frac{t}{2} \mathcal{H}} \bar{\Pi}_{n}\right| \Psi\left(\mathbf{u}_{N}\right)\right\rangle \\
& =\sum_{\boldsymbol{\lambda}^{L, R} \backslash \mathbf{n} \subseteq\left\{(\mathcal{M}-n)^{N}\right\}} S_{\boldsymbol{\lambda}^{L}}\left(\mathbf{v}_{N}^{-2}\right) S_{\boldsymbol{\lambda}^{R}}\left(\mathbf{u}_{N}^{2}\right) G\left(\boldsymbol{\lambda}^{L} ; \boldsymbol{\lambda}^{R} \mid t\right) \\
& =\sum_{K=0}^{\infty} \frac{(t / 2)^{K}}{K !} \sum_{\boldsymbol{\lambda}^{L, R} \backslash \mathbf{n} \subseteq\left\{(\mathcal{M}-n)^{N}\right\}} S_{\boldsymbol{\lambda}^{L}}\left(\mathbf{v}_{N}^{-2}\right) S_{\boldsymbol{\lambda}^{R}}\left(\mathbf{u}_{N}^{2}\right) \mathfrak{G}\left(\boldsymbol{\mu}^{L} ; \boldsymbol{\mu}^{R} \mid K\right)
\end{aligned}
$$

is calculated with the help of Eqs. (5) and (9) [19]. In the above formulas $\mathfrak{G}\left(\boldsymbol{\mu}^{L} ; \boldsymbol{\mu}^{R} \mid K\right)$ is given either by (28) or (34), $\mathbf{u}_{N}$ and $\mathbf{v}_{N}$ stand for an arbitrary parametrization, and two independent summations over $\boldsymbol{\lambda}^{L, R}=\boldsymbol{\mu}^{L, R}-\boldsymbol{\delta}_{N}$ are analogous to those in (36).

Substituting (31) into (53), we obtain the transition amplitude in the form, 18]:

$$
\begin{array}{r}
\left\langle\Psi\left(\mathbf{v}_{N}\right)\left|\bar{\Pi}_{n} e^{-t \mathcal{H}} \bar{\Pi}_{n}\right| \Psi\left(\mathbf{u}_{N}\right)\right\rangle=\frac{1}{(M+1)^{N} N !} \sum_{s_{1}, \ldots, s_{N}=0}^{M} e^{t \sum_{m=0}^{N} \cos \phi_{m}} \\
\times\left|\mathcal{V}\left(e^{i \phi_{N}}\right)\right|^{2} \mathcal{P}_{\mathcal{M}-n}\left(\mathbf{v}^{-2}, e^{i \phi}\right) \mathcal{P}_{\mathcal{M}-n}\left(e^{-i \phi}, \mathbf{u}^{2}\right)
\end{array}
$$

where $\mathcal{P}_{\mathcal{M}-n}$ is the sum $(36)$.

Let us consider the expansion of the transition amplitude in the case when $\mathbf{u}_{N}^{2}=$ $\mathbf{v}_{N}^{2}=(1,1, \ldots, 1)$. From $52-54$ we obtain for the $K^{\text {th }}$ term:

$$
\begin{aligned}
& \left\langle\Psi(\mathbf{1})\left|\bar{\Pi}_{n}(-\mathcal{H})^{K} \bar{\Pi}_{n}\right| \Psi(\mathbf{1})\right\rangle \\
& =\sum_{\boldsymbol{\lambda}^{L, R} \backslash \mathbf{n} \subseteq\left\{(\mathcal{M}-n)^{N}\right\}} S_{\boldsymbol{\lambda}^{L}}(\mathbf{1}) S_{\boldsymbol{\lambda}^{R}}(\mathbf{1})\left|P_{K}\left(\boldsymbol{\mu}^{R} \rightarrow \boldsymbol{\mu}^{L}\right)\right|,
\end{aligned}
$$

where $\left|P_{K}\left(\boldsymbol{\mu}^{R} \rightarrow \boldsymbol{\mu}^{L}\right)\right|$ are integers given by $(29)$, and the values of the Schur functions $S_{\boldsymbol{\lambda}^{L}}(\mathbf{1})$ and $S_{\boldsymbol{\lambda}^{R}}(\mathbf{1})$ are given by (45). If we put $\mathbf{u}_{N}^{2}=\mathbf{v}_{N}^{2}=(1,1, \ldots, 1)$ in (55), a representation alternative to 56 is obtained:

$$
\begin{aligned}
\left\langle\Psi(\mathbf{1})\left|\bar{\Pi}_{n}(-\mathcal{H})^{K} \bar{\Pi}_{n}\right|\right. & \Psi(\mathbf{1})\rangle=\frac{1}{(M+1)^{N}} \sum_{\left\{\boldsymbol{\phi}_{N}\right\}}\left(2 \sum_{m=1}^{N} \cos \phi_{m}\right)^{K} \\
& \times\left|\mathcal{V}\left(e^{i \boldsymbol{\phi}_{N}}\right)\right|^{2} \mathcal{P}_{\mathcal{M}-n}\left(\mathbf{1}, e^{i \boldsymbol{\phi}}\right) \mathcal{P}_{\mathcal{M}-n}\left(e^{-i \boldsymbol{\phi}}, \mathbf{1}\right) .
\end{aligned}
$$

Since left-hand sides of (56) and (57) coincide, we obtain the following equality of two sums:

$$
\begin{array}{r}
\frac{1}{(M+1)^{N}} \sum_{\left\{\phi_{N}\right\}}\left(2 \sum_{m=1}^{N} \cos \phi_{m}\right)^{K}\left|\mathcal{V}\left(e^{i \phi_{N}}\right) \mathcal{P}_{\mathcal{M}-n}\left(\mathbf{1}, e^{i \phi}\right)\right|^{2} \\
=\sum_{\boldsymbol{\lambda}^{L, R} \backslash \mathbf{n} \subseteq\left\{(\mathcal{M}-n)^{N}\right\}} S_{\boldsymbol{\lambda}^{L}}(\mathbf{1}) S_{\boldsymbol{\lambda}^{R}}(\mathbf{1})\left|P_{K}\left(\boldsymbol{\mu}^{R} \rightarrow \boldsymbol{\mu}^{L}\right)\right|
\end{array}
$$


In other words, the trigonometric sum in left-hand side of (58) acquires an integer value expressed by the sum in right-hand side of (58).

Summarising the graphical representations of functions involved in right-hand side of (56) we can give the graphical representation of the $K^{\text {th }}$ term of the transition amplitude in terms of nests of self-avoiding lattice paths. The first steps particles are doing according the lock step rules starting from sites $C_{i}$ and finishing at accessible intermediate positions $\boldsymbol{\mu}^{R}$. The number of these nests is $S_{\boldsymbol{\lambda}^{R}}(\mathbf{1})$. The next $K$ steps particles are doing according to the random turns rules starting from sites $\boldsymbol{\mu}^{L}$ and terminating at $\boldsymbol{\mu}^{R}$. The number of these nests is $\left|P_{K}\left(\boldsymbol{\mu}^{R} \rightarrow \boldsymbol{\mu}^{L}\right)\right|$. The final steps are made again by the lock step rules from $\boldsymbol{\mu}^{R}$ up to $B_{i}$, and the number of these nests is $S_{\boldsymbol{\lambda}^{R}}(\mathbf{1})$. An example of the described nest of lattice paths is depicted in Fig. 8 . Furthermore, two independent summations over intermediate positions $\boldsymbol{\mu}^{L, R}$ are meant in right-hand side of (56). To obtain the transition amplitude we have to sum up over all intermediate steps $K(54)$.

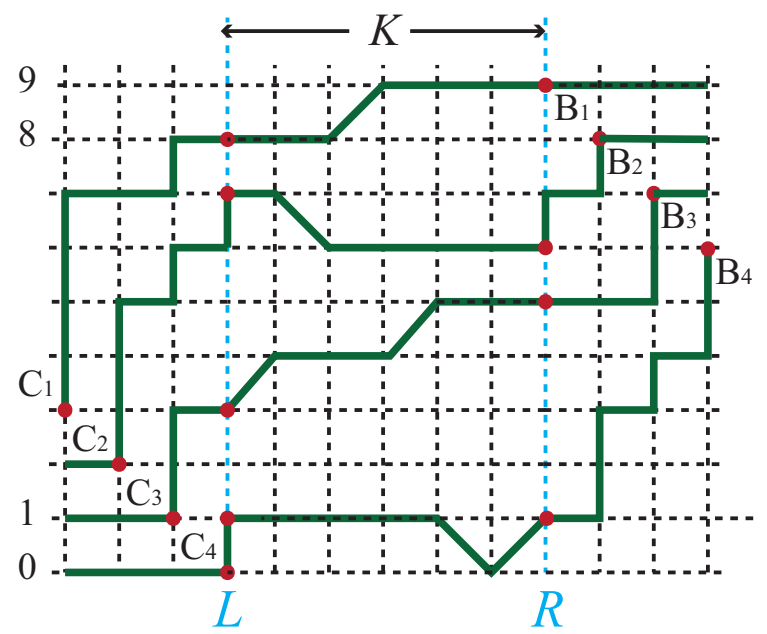

Figure 8: Nest of paths contributing to $\left\langle\Psi(\mathbf{1})\left|\bar{\Pi}_{1}(-\mathcal{H})^{K} \bar{\Pi}_{1}\right| \Psi(\mathbf{1})\right\rangle$.

Eventually, we use (55) and obtain the persistence of ferromagnetic string $\mathcal{T}\left(\boldsymbol{\theta}_{N}^{\mathrm{g}}, n, t\right)$ (15):

$$
\begin{array}{r}
\mathcal{T}\left(\boldsymbol{\theta}_{N}^{\mathrm{g}}, n, t\right)=\frac{1}{\mathcal{N}^{2}\left(\boldsymbol{\theta}_{N}^{\mathrm{g}}\right)(M+1)^{N}} \sum_{\left\{\boldsymbol{\theta}_{N}\right\}} e^{-t\left(E_{N}\left(\boldsymbol{\theta}_{N}\right)-E_{N}\left(\boldsymbol{\theta}_{N}^{\mathrm{g}}\right)\right)} \\
\times\left|\mathcal{V}_{N}\left(e^{i \boldsymbol{\theta}_{N}}\right) \mathcal{P}_{\mathcal{M}-n}\left(e^{-i \boldsymbol{\theta}_{N}}, e^{i \boldsymbol{\theta}_{N}^{\mathrm{g}}}\right)\right|^{2}
\end{array}
$$

where summation is over all independent solutions of the Bethe equations (10). The $\operatorname{sum} \mathcal{P}_{\mathcal{M}-n}\left(e^{-i \boldsymbol{\theta}_{N}}, e^{i \boldsymbol{\theta}_{N}^{\mathrm{g}}}\right)$ is given by 36 on the solutions to Eqs. 10 , and $\mathcal{N}^{2}\left(\boldsymbol{\theta}_{N}^{\mathrm{g}}\right)$ is the squared norm of the ground state: $\mathcal{N}^{2}\left(\boldsymbol{\theta}_{N}^{\mathrm{g}}\right)=\left\langle\Psi\left(\boldsymbol{\theta}_{N}^{\mathrm{g}}\right) \mid \Psi\left(\boldsymbol{\theta}_{N}^{\mathrm{g}}\right)\right\rangle$, where $\boldsymbol{\theta}_{N}^{\mathrm{g}}$ are given by (14). 


\section{Conclusion}

The representation of the Schur functions in terms of nests of self-avoiding lattice paths of lock step type, as well as the representation of the averages (28) in terms of random turns walks, made it possible for us to represent the correlation function of persistence of ferromagnetic string (15) in the graphical way. Equation (58) is the main technical result of the paper obtained by means of the graphical approach developed.

\section{Acknowledgement}

The research described has been partially supported by RSF grant no. 16-11-10218.

\section{References}

[1] Stanley, R., Enumerative Combinatorics, vol. 2. Cambridge University Press, Cambridge, 1999.

[2] Williams, C., Explorations in Quantum Computing. Springer, 2010.

[3] Fama, E., Random Walks in Stock Market Prices. Financial Analysts Journal 51, 75 (1995).

[4] Sneppen, K., Models of Life. Cambridge University Press, 2014.

[5] Contributions to Mathematical Psychology, Psychometrics, and Methodology. Eds., G. Fischer, D. Laming. Springer, 2012.

[6] Redner, S., A Guide to First-Passage Processes. Cambridge University Press, 2001.

[7] Renshaw, E., Stochastic Population Processes. Oxford University Press, 2011.

[8] Fisher, M., Walks, Walls, Wetting and Melting. J. Stat. Phys. 34, 667 (1984).

[9] Forrester, P., Exact Solution of the Lock Step Model of Vicious Walkers. J. Phys. A.: Math. Gen. 23, 1259 (1990).

[10] Nagao, T., Forrester, P., Vicious Random Walkers and a Discretization of Gaussian Random Matrix Ensembles. Nucl. Phys. B 620, 551 (2002).

[11] Guttmann, A., Owczarek, A., Viennot, X., Vicious Walkers and Young Tableaux I: Without Walls. J. Phys. A: Math. Gen. 31, 8123 (1998).

[12] Krattenthaler, C., Guttmann, A., Viennot, X., Vicious Walkers, Friendly Walkers and Young Tableaux: II. With a Wall. J. Phys. A: Math. Gen. 33, 8835 (2000).

[13] Katori, M., Tanemura, H., Scaling Limit of Vicious Walks and Two-Matrix Model. Phys. Rev. E 66, 011105 (2002). 
[14] Bogoliubov, N., XX Heisenberg Chain and Random Walks. J. Math. Sci. 138, 5636 (2006).

[15] Macdonald, I., Symmetric Functions and Hall Polynomials. Clarendon Press, 1995.

[16] Faddeev, L., Quantum Completely Integrable Models in Field Theory. Sov. Sci. Rev. C: Math. Phys. 1, 107 (1980).

[17] Korepin, V., Bogoliubov, N., Izergin, A., Quantum Inverse Scattering Method and Correlation Functions. Cambridge University Press, Cambridge, 1993.

[18] Bogoliubov, N., Malyshev, C., Correlation Functions of XX0 Heisenberg Chain, q-Binomial Determinants, and Random Walks. Nucl. Phys. B 879, 268 (2014).

[19] Bogoliubov, N., Malyshev, C., Integrable Models and Combinatorics. Russian Math. Surveys 70, 789 (2015).

[20] Bogoliubov, N., Malyshev, C., Multi-Dimensional Random Walks and Integrable Phase Models. J. Math. Sci. (New York) 224, 199 (2017).

[21] Bogoliubov, N., Malyshev, C., Zero Range Process and Multi-Dimensional Random Walks. SIGMA 13, 056 (2017).

[22] Jin, B.-Q., Korepin, V. E., Entanglement, Toeplitz Determinants and FisherHartwig Conjecture. J. Stat. Phys. 116, 79 (2004).

[23] Bogoliubov, N., Malyshev, C., Correlation Functions of the XX Heisenberg Magnet and Random Walks of Vicious Walkers. Theor. Math. Phys. 159, 563 (2009).

[24] Fulton, W., Young tableaux. With applications to representation theory and geometry, London Math. Soc. Stud. Texts, vol. 35, Cambridge Univ. Press, Cambridge, 1997.

[25] Gross, D. J., Witten, E., Possible Third-Order Phase Transition in the Large-N Lattice Gauge Theory. Phys. Rev. D 21, 446 (1980).

[26] Johansson, K., Unitary Random Matrix Model. Mathematical Research Letters 5, 63 (1998). 